\title{
Anastomosing hemangioma: The first case report in the bladder
}

\author{
LU JIN ${ }^{1,2^{*}}$, JIAJU LIU ${ }^{2,3^{*}}$, YIFAN LI ${ }^{1,2}$, SHUOLEI SUN ${ }^{2}$, XIANGMING MAO ${ }^{2}$, \\ SHANGQI YANG ${ }^{2}$ and YONGQING LAI ${ }^{2}$ \\ ${ }^{1}$ The Second Clinical College, College of Clinical Medicine, Anhui Medical University, Hefei, Anhui 230032; \\ ${ }^{2}$ Department of Urology, Peking University Shenzhen Hospital, Shenzhen, Guangdong 518036; ${ }^{3}$ Department \\ of Clinical Medicine, Shantou University Medical College, Shantou, Guangdong 515041, P.R. China
}

Received July 3, 2015; Accepted November 19, 2015

DOI: $10.3892 / \mathrm{mco} .2015 .699$

\begin{abstract}
A rare neoplasm, termed 'anastomosing hemangioma' $(\mathrm{AH})$, has been previously described in the genitourinary tract. To date, 29 cases of AH have been reported in the literature, and the case reported in the present study is, to the best of our knowledge, the first AH described in the urinary bladder. It is essential to distinguish $\mathrm{AH}$ from malignant vascular tumors due to the different treatments required and the prognosis. The patient in this case study was asymptomatic, with a neoplasm of $\sim 1 \mathrm{~cm}$ within the right wall of the bladder. Pathologically, the lesion was predominantly composed of a structure comprising a small vascular cavity and irregularly fenestrated anastomosing vascular channels, with no clear atypia of the endothelial cells.
\end{abstract}

\section{Introduction}

Primary benign vascular lesions are more frequently located in the skin and subcutaneous soft tissues compared with the viscera, particularly the genitourinary system. In 2009, Montgomery and Epstein (1) described for the first time a novel subtype of benign vascular lesion, which was termed an 'anastomosing hemangioma' (AH), of the genitourinary tract (kidney and testis). Subsequently, several cases of AH were reported in the adrenal gland, ovary, liver and gastrointestinal tract (2-4). Although AH occurs relatively infrequently, an incidence of $\mathrm{AH}$ in the bladder has arisen during our medical researches. In the present case study, to the best of our knowl-

Correspondence to: Professor Yongqing Lai or Professor Shangqi Yang, Department of Urology, Peking University Shenzhen Hospital, 1120 Lianhua Road, Shenzhen, Guangdong 518036, P.R. China

E-mail: yqlord@163.com

E-mail: yangshangqi88@aliyun.com

*Contributed equally

Key words: anastomosing hemangioma, genitourinary tract, bladder neoplasm edge, the first case of AH in the bladder has been reported, accompanied by a review of the literature on $\mathrm{AH}$.

\section{Case report}

The patient was a 46-year-old Chinese man who had a history of renal calculus and who underwent extracorporeal shock wave lithotripsy twice (in 2009 and 2013). During a medical examination (in March 2015), abdominal ultrasonography revealed that, within the right wall of the bladder, there was a substantial low echo nodule, measuring $14 \times 11 \mathrm{~mm}$, which was papillary in shape and protruded into the bladder lumen. However, the patient remained asymptomatic, and neither a physical examination nor a standard laboratory examination revealed any noteworthy phenomena. The lesion was further investigated using a contrast-enhanced computerized tomography scan, and the results confirmed the identification of an oval, sharply defined lesion within the right wall of the bladder, which exhibited a marked homogeneous enhancement (Figs. 1 and 2). This indicated the possibility that the lesion may have been a vascular tumor. Subsequently, cystoscopy was performed, and a sample of the lesion tissue was removed prior to pathological examination. No characteristic tumor tissue was identified on the mucosal surface of the bladder, although congestive mucosa was observed on the right wall of the bladder, and the pathological results revealed partial nuclear atypia.

On the basis of the results of the imaging and the pathological findings, the patient finally underwent a partial cystectomy (in April 2015). The pathological diagnosis was given as 'intramural AH of the bladder'. The gross pathological examination revealed a well-circumscribed, russet-colored tissue, bearing a fish/meat-like, gray-brown cut surface. The microscopic examination revealed that the tumor tissue was lobulated, alternating with sections of muscle. Furthermore, the structure of the lesion predominantly comprised a small vascular cavity with irregularly fenestrated, anastomosing vascular channels, with no obvious atypia of the endothelial cells (Fig. 3). These anatomical features supported the diagnosis of $\mathrm{AH}$ of the bladder.

\section{Discussion}

Most bladder tumors are epithelial, and particularly urothelial, in origin, and non-urothelial neoplasms occur very 


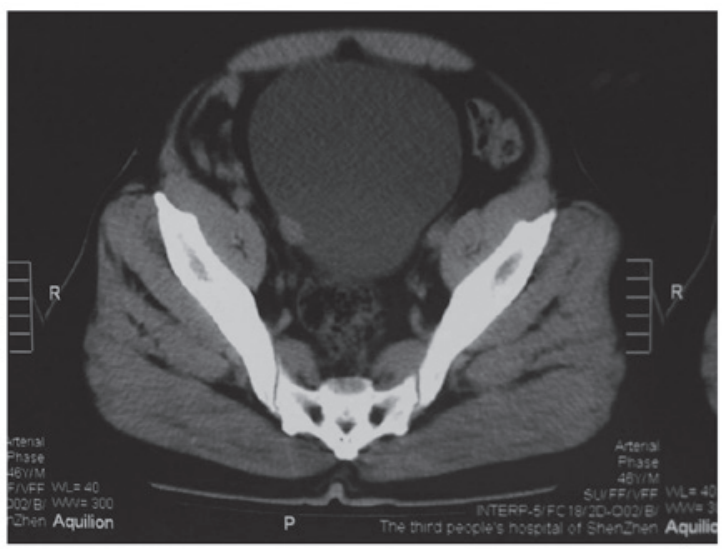

Figure 1. Non-enhanced axial computed tomography image, showing a sharply defined lesion within the right wall of the bladder.

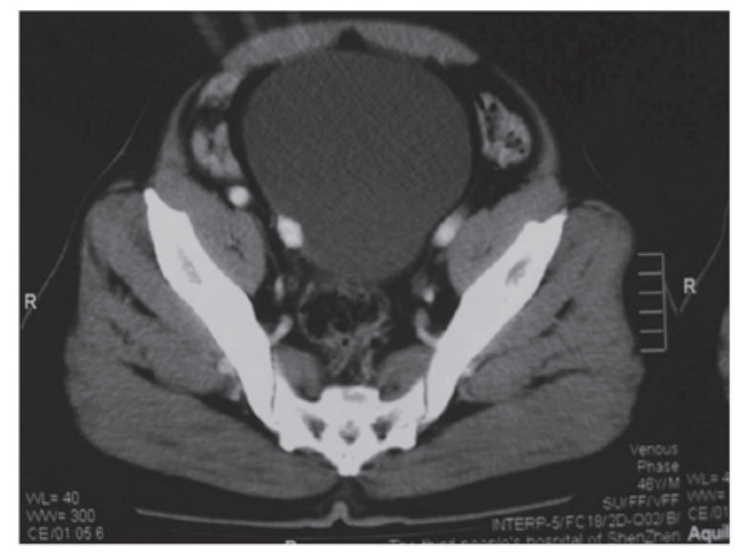

Figure 2. Contrast-enhanced axial computerized tomography image, showing a marked homogenous enhancement.

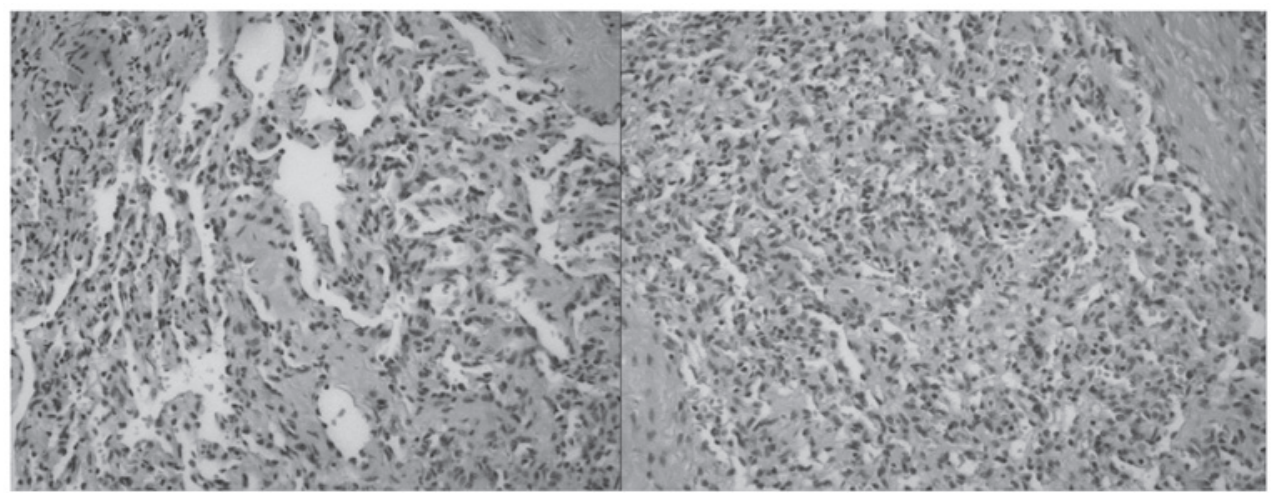

Figure 3. Using hematoxylin and eosin staining, the pathological results revealed that the structure of the tissue is predominantly composed of small vascular cavities and irregularly fenestrated, anastomosing vascular channels, with no clear atypia of the endothelial cells (magnification, x200).

rarely in the bladder. In addition, sarcoma accounts for the majority of the non-urothelial neoplasms (5); hemangioma is extremely rare in the urinary bladder. Non-urothelial bladder tumors, including squamous cell carcinoma, adenocarcinoma and sarcoma, usually present a diagnostic challenge. In the present study, to the best of our knowledge, the first identified case of $\mathrm{AH}$ in the bladder has been reported, which has increased the diagnostic challenge of intramural tumors of the bladder. Hemangioma is very common in the skin and subcutaneous soft tissue, and occasionally occurs in viscera, and Montgomery and Epstein (1) first described $\mathrm{AH}$ in the kidney and testis.

In the present case study, an AH located within the bladder wall has been described, to the best of our knowledge for the first time. To date, 29 cases of AH have been reported in the English literature, a majority of them located in the kidney (1-4,6-11). The immunohistochemical findings indicated that CD34 was immunoreactive in all the tissues examined. Generally, in the kidney, most identified lesions have occurred singly, of median size $2 \mathrm{~cm}$ (range, 0.6-5 cm), and were associated with end-stage renal disease (7). Additionally, a majority of the patients were men, exhibiting no characteristic symptoms. Imageological examinations proved incapable of differentiating the $\mathrm{AH}$ from aggressive tumors, and most of the patients with renal AH underwent a nephrectomy (12). A number of the risk factors remained unidentified, although, according to the cases reported, being of the male gender is a risk factor.

Although the occurrence of $\mathrm{AH}$ is uncommon in the genitourinary tract, pathologists and doctors ought to take it into consideration in making the differential diagnosis, in order to avoid misdiagnosis. In the present case study, it would have been easy to have mistaken the AH as invasive, high-grade, urothelial carcinoma. A differential diagnosis of AH is predominantly made with malignant vascular tumors, including angiosarcoma, which is usually an aggressive tumor characterized by infiltrative growth, clear cytological atypia, high cellularity and poor prognosis. Otherwise, in forming a diagnosis, it is essential to distinguish the $\mathrm{AH}$ from other intramural tumors, including paragangliomas and bladder sarcoma. The pathological finding that $\mathrm{AH}$ is composed of a small vascular cavity with irregularly fenestrated, anastomosing vascular channels allows the correct diagnosis to be made.

According to the previously reported cases, upon microscopical examination, $\mathrm{AH}$ is typically characterized by numerous thin-walled, vascular channels, which grow according to an anastomosing pattern, and which lack distinct endothelial atypia or multilayering (1). Certain variants of glomus tumors should also be taken into consideration, for they too possess an abundant vasculature network (12). The majority of the clinical follow-up of the AH occurs in a 
time-frame of $\sim 3$ months to 3 years, the longest reported case being of 13 years (12). Although $\mathrm{AH}$ is a type of tumor which was considered to be benign, given that the most important differential diagnosis of $\mathrm{AH}$ is aggressive angiosarcoma and that imageological examinations are unable to differentiate the AH from aggressive tumors, the majority of patients with renal $\mathrm{AH}$ have undergone a nephrectomy, or partial nephrectomy (12). However, it must be recognized that nephrectomy is an excessive treatment for patients with renal $\mathrm{AH}$. The case reported in the present study was the first identified case of AH occurring in the bladder, and the patient underwent partial cystectomy.

Clinically, abdominal B-ultrasonography has been used for the initial examination of patients with bladder cancer, and has been selected for the majority of bladder tumors due to its non-invasive properties, speediness and the ease of convenience. The bladder mass of the patient described in the present case study was initially identified using ultrasonography, without any discomfort for the patient, who is recovering well one month following the surgery. This case has highlighted that the differential diagnosis of intramural neoplasms in the bladder is vitally important for managing the different treatment required, and for the prognosis.

In conclusion, in the present case study, an $\mathrm{AH}$ located in the wall of bladder was identified, to the best of our knowledge for the first time. In view of the limited number of reported cases, there is no 'gold standard' treatment for AH, and men are considered to be at greater risk compared with women. It is important to distinguish AH from malignant vascular tumors, since the required therapeutic approach, and the prognosis between them, may differ substantially.

\section{Acknowledgements}

This study was supported by the National Natural Science Foundation of China (no. 81101922), Science and Technology Development Fund Project of Shenzhen (nos. JCYJ20130402114702124 and JCYJ20150403091443329) and the fund of the Guangdong Key medical subject.

\section{References}

1. Montgomery E and Epstein JI: Anastomosing hemangioma of the genitourinary tract: A lesion mimicking angiosarcoma. Am J Surg Pathol 33: 1364-1369, 2009.

2. Ross M, Polcari A, Picken M, Sankary H and Milner J: Anastomosing hemangioma arising from the adrenal gland. Urology 80: e27-e28, 2012.

3. Kryvenko ON, Gupta NS, Meier FA, Lee MW and Epstein JI: Anastomosing hemangioma of the genitourinary system: Eight cases in the kidney and ovary with immunohistochemical and ultrastructural analysis. Am J Clin Pathol 136: 450-457, 2011.

4. Lin J, Bigge J, Ulbright TM and Montgomery E: Anastomosing hemangioma of the liver and gastrointestinal tract: An unusual variant histologically mimicking angiosarcoma. Am J Surg Pathol 37: 1761-1765, 2013.

5. Dahm P and Gschwend JE: Malignant non-urothelial neoplasms of the urinary bladder: A review. Eur Urol 44: 672-681, 2003.

6. Chou S, Subramanian V, Lau HM and Achan A: Renal anastomosing hemangiomas with a diverse morphologic spectrum: Report of two cases and review of literature. Int J Surg Pathol 22: 369-373, 2014.

7. Zhao M, Li C, Zheng J and Sun K: Anastomosing hemangioma of the kidney: A case report of a rare subtype of hemangioma mimicking angiosarcoma and review of the literature. Int J Clin Exp Pathol 6: 757-765, 2013.

8. Tao LL, Dai Y, Yin W and Chen J: A case report of a renal anastomosing hemangioma and a literature review: An unusual variant histologically mimicking angiosarcoma. Diagn Pathol 9: 159, 2014.

9. Tahir $\mathrm{M}$ and Folwell A: Anastomosing haemangioma of kidney: A rare subtype of vascular tumour of the kidney mimicking angiosarcoma. ANZ J Surg, 2014 (Epub ahead of print).

10. Wetherell DR, Skene A, Manya K, Manecksha RP, Chan Y and Bolton DM: Anastomosing haemangioma of the kidney: A rare morphological variant of haemangioma characteristic of genitourinary tract location. Pathology 45: 193-196, 2013.

11. Tran TA and Pernicone P: Anastomosing hemangioma with fatty changes of the genitourinary tract: A lesion mimicking angiomyolipoma. Cent European J Urol 65: 40-42, 2012.

12. Heidegger I, Pichler R, Schäfer G, Zelger B, Zelger B, Aigner F, Bektic J and Horninger W: Long-term follow up of renal anastomosing hemangioma mimicking renal angiosarcoma. Int J Urol 21: 836-838, 2014. 